\title{
Diffusioosmosis and Electroosmosis of Electrolyte Solutions in Fibrous Porous Media
}

\author{
Huan J. Keh ${ }^{1}$ and Yeu K. Wei \\ Department of Chemical Engineering, National Taiwan University, Taipei 106-17, Taiwan, ROC
}

Received January 2, 2002; accepted May 10, 2002

The steady diffusioosmotic and electroosmotic flows of an electrolyte solution in the fibrous porous medium constructed by a homogeneous array of parallel charged circular cylinders are analyzed under conditions of small Peclet and Reynolds numbers. The imposed electrolyte concentration gradient or electric field is constant and can be oriented arbitrarily with respect to the axes of the cylinders. The thickness of the electric double layers surrounding the cylinders is assumed to be small relative to the radius of the cylinders and to the gap width between two neighboring cylinders, but the polarization effect of the diffuse ions in the double layers is incorporated. Through the use of a unit cell model, the appropriate equations of conservation of the electrochemical potential energies of ionic species and the fluid momentum are solved for each cell, in which a cylinder is envisaged to be surrounded by a coaxial shell of the fluid. Analytical expressions for the diffusioosmotic and electroosmotic velocities of the bulk electrolyte solution as functions of the porosity of the ordered array of cylinders are obtained in closed form for various cases. Comparisons of the results of the cell model with different conditions at the outer boundary of the cell are made. In the limit of maximum porosity, these results can be interpreted as the diffusiophoretic and electrophoretic velocities of an isolated circular cylinder caused by the imposed electrolyte concentration gradient or electric field. (c) 2002 Elsevier Science (USA)

Key Words: diffusioosmosis and diffusiophoresis; electroosmosis and electrophoresis; charged circular cylinder; unit cell model; thin but polarized double layer.

\section{INTRODUCTION}

The electrokinetic phenomena in porous media with charged surfaces are of much fundamental and practical interest in various fields of science and engineering. In general, driving forces for the flow of electrolyte solutions in a small pore with a charged wall include dynamic pressure differences between the two ends of the pore (a streaming potential is developed as a result of the requirement of no net electric current) and tangential electric fields that interact with the electric double layer adjacent to the pore wall (electroosmosis). Problems of electrokinetic flow

\footnotetext{
${ }^{1}$ To whom correspondence and reprint requests should be addressed. Fax: (886) 2 23623040. E-mail: huan@ccms.ntu.edu.tw.
}

caused by these driving forces were studied extensively in the past (1-3). For an electrolyte solution subjected to a uniformly applied electric field $\mathbf{E}^{\infty}$ in the direction parallel to a plane wall, the electroosmotic velocity outside the double layer is given by the well-known Helmholtz equation

$$
\mathbf{U}_{0}=-\frac{\varepsilon \zeta}{4 \pi \eta} \mathbf{E}^{\infty}
$$

where $\varepsilon / 4 \pi$ is the fluid permittivity, $\eta$ is the fluid viscosity, and $\zeta$ is the zeta potential of the wall.

Another driving force for the electrokinetic flow in a micropore, which has commanded less attention, involves tangential concentration gradients of an ionic solute that interacts with the charged pore wall. This solute-wall interaction is electrostatic in nature and its range is the electric double layer adjacent to the wall. The fluid motion associated with this mechanism, known as "diffusioosmosis" (and in contrast to the diffusiophoresis of colloidal particles in prescribed solute concentration gradients), has been discussed for electrolyte solutions near a plane wall $(3,4)$ and inside a straight capillary (5-8). For a solution of a symmetrically charged binary electrolyte with a constant concentration gradient $\nabla n^{\infty}$ along a plane wall, the diffusioosmotic velocity outside the double layer can be expressed as (4)

$$
\mathbf{U}_{0}=-\frac{\varepsilon \zeta}{4 \pi \eta}\left(\lambda+\bar{\zeta}^{-1} \ln \cosh \bar{\zeta}\right) \frac{k T}{Z e} \nabla \ln n^{\infty}
$$

with

$$
\begin{aligned}
& \lambda=\frac{D_{2}-D_{1}}{D_{2}+D_{1}}, \\
& \bar{\zeta}=\frac{Z e \zeta}{4 k T} .
\end{aligned}
$$

Here, $D_{1}$ and $D_{2}$ are the diffusion coefficients of the anion and cation, respectively, $Z$ is the absolute value of the valences of ions, $e$ is the elementary electric charge, $k$ is Boltzmann's constant, and $T$ is the absolute temperature. Equation [2] predicts that the electrolyte solution can flow toward or away from the 
end of higher concentration, depending on the combination of the diffusion coefficients of the ions and the zeta potential of the wall. For the special case of $D_{2}=D_{1}$ (or $\lambda=0$ ), the fluid flow (due to chemiosmosis only) is in the direction of decreasing electrolyte concentration regardless of the sign of $\zeta$ and the fluid mobility is a monotonically increasing function of the magnitude of $\zeta$.

The formulas for the electroosmotic and diffusioosmotic velocities of bulk electrolyte solutions parallel to a charged plane wall (given by Eqs. [1] and [2]) can also be applied to the corresponding flow in straight capillaries when the thickness of the double layer adjacent to the capillary wall is small compared with the capillary radius. However, the capillary model of porous media is not a realistic model for either granular or fibrous systems, for it does not allow for the convergence and divergence of flow channels. For electroosmotic or diffusioosmotic flow within beds of particles, fibrous matrices, or microporous membranes, it is usually necessary to account for the effects of pore geometry, tortuosity, etc. To avoid the difficulty of the complex geometry appearing in beds of particles, unit cell models were often employed to predict these effects on the relative motions between a granular bed and the bulk fluid. These models involve the concept that a bed of identical particles can be divided into a number of identical cells, one particle occupying each cell at its center. The boundary value problem for multiple particles is thus reduced to the consideration of the behavior of a single particle and its bounding envelope. The most acceptable of these models with various boundary conditions at the outer (virtual) surface of a cell are the "free-surface" model of Happel $(9,10)$ and the "zero-vorticity" model of Kuwabara (11). In the past, the unit cell models were used by many researchers to predict various transport properties, such as the mean sedimentation rate $(12,13)$ and electrophoretic mobility (14-20) in homogeneous suspensions of charged spherical particles as well as the electroosmotic mobility of electrolyte solutions in fibrous porous media (21-24). Recently, using the cell models, the present authors derived analytical expressions for the mean diffusiophoretic velocity of a swarm of dielectric spheres (25), which can also apply for the diffusioosmotic velocity of electrolyte solutions within a fixed bed of charged spheres.

In this work, the Happel and Kuwabara cell models are used to obtain analytical expressions for the diffusioosmotic and electroosmotic velocities of a solution of a symmetrically charged electrolyte subjected to a uniformly imposed and arbitrarily oriented concentration gradient or electric field within a homogeneous array of parallel charged circular cylinders. The thickness of the electric double layers is assumed to be small relative to the radius of the cylinders and to the gap width between two neighboring cylinders, but the effect of polarization of the mobile ions in the thin double layers is taken into account. The analytical solutions in closed form obtained with the cell models enable the diffusioosmotic and electroosmotic velocities of the bulk fluid to be predicted as functions of the porosity of the fibrous porous medium for various cases.

\section{ANALYSIS OF DIFFUSIOOSMOSIS}

We consider the diffusioosmotic flow of a fluid solution of a symmetrically charged electrolyte in a fibrous porous medium constructed by a homogeneous array of parallel identical circular cylinders at the steady state. The cylinders are charged uniformly on the surfaces, and the fluid is assumed to be incompressible and Newtonian. The thin electric double layers surrounding the cylinders can be polarized but do not overlap with one another. The applied electrolyte concentration gradient $\nabla n^{\infty}$ (where $n^{\infty}$ is the linear concentration distribution of the electrolyte prescribed in the absence of the cylinders) can be taken as a combination of its transverse and longitudinal components with respect to the orientation of the cylinders,

$$
\nabla n^{\infty}=\left(\nabla n^{\infty}\right)_{\mathrm{t}} \mathbf{e}_{x}+\left(\nabla n^{\infty}\right)_{1} \mathbf{e}_{z}
$$

where $\mathbf{e}_{x}$ and $\mathbf{e}_{z}$ are the unit vectors in the directions normal and parallel, respectively, to the axes of the cylinders. Then, the problem can be divided into two due to the linearity and they will be separately solved. The overall diffusioosmotic velocity of the bulk fluid can be obtained by the vectorial addition of the two-component results.

First, we consider the diffusioosmosis of the fluid solution due to the transverse component of the electrolyte gradient. The bulk diffusioosmotic velocity of the solution caused by the gradient $\left(\nabla n^{\infty}\right)_{\mathrm{t}}$ can be written as $-U_{\mathrm{t}} \mathbf{e}_{x}$. As shown in Fig. 1, we employ a unit cell model in which each dielectric cylinder of radius $a$ is surrounded by a coaxial circular cylindrical shell of the fluid solution having an outer radius of $b$ such that the fluid/cell volume ratio is equal to the porosity $1-\varphi$ of the fiber matrix; viz. $\varphi=(a / b)^{2}$. The origin of the polar coordinate system $(\rho, \phi)$ is taken at the axis of the cylinder and the polar axis $\phi=0$ points toward the positive $x$ direction. Obviously, the two-dimensional problem for each cell is symmetric about the $x$ axis. Our

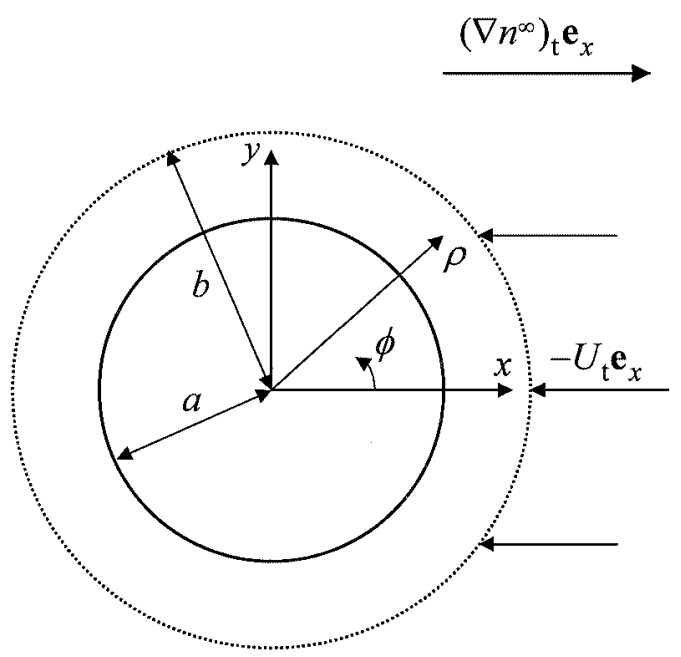

FIG. 1. Geometrical sketch for the transverse diffusioosmosis of an electrolyte solution around a circular cylinder in a coaxial cylindrical cell. 
objective is to determine the bulk fluid velocity induced by the diffusioosmotic driving force in the limit of small Peclet and Reynolds numbers.

The fluid phase in a cell can be divided into two regions: an "inner" region defined as the thin double layer surrounding the cylinder and an "outer" region defined as the remainder of the fluid which is neutral. In the outer region, the equations of conservation of each ionic species and the fluid momentum are the Laplace equation $(26,27)$

$$
\nabla^{2} \mu_{m}=0, \quad m=1,2,
$$

and the Stokes equation for viscous two-dimensional flows

$$
\nabla^{2}\left(\nabla^{2} \Psi\right)=0 .
$$

In Eq. [6], $\mu_{m}(\rho, \phi)$ is the electrochemical potential energy of species $m$ defined by

$$
\mu_{m}=\mu_{m}^{0}+k T \ln n_{m}+z_{m} e \Phi,
$$

where $\mu_{m}^{0}$ is a constant, $n_{m}(\rho, \phi)$ and $z_{m}$ are the concentration and valence, respectively, of type $m$ ions, and $\Phi(\rho, \phi)$ is the electric potential. The subscript $m$ equal to 1 and 2 refers to the anion and cation, respectively, so $-z_{1}=z_{2}=Z$. Note that, in the outer region, $n_{1}=n_{2}=n$, and both $n$ and $\Phi$ also satisfy Laplace's equation. In Eq. [7], $\Psi(\rho, \phi)$ is the stream function related to the $\rho$ and $\phi$ components of the velocity field by

$$
\begin{aligned}
& v_{\rho}=\frac{1}{\rho} \frac{\partial \Psi}{\partial \phi}, \\
& v_{\phi}=-\frac{\partial \Psi}{\partial \rho} .
\end{aligned}
$$

The governing Eqs. [6] and [7] in the outer region satisfy the following boundary conditions at the cylinder surface (outer edge of the thin double layer) obtained by solving for the electrochemical potentials and fluid velocity in the inner region and using a matching procedure to ensure a continuous solution in the whole fluid phase $(26,27)$ :

$$
\begin{aligned}
\rho=a: \frac{\partial \mu_{m}}{\partial \rho} & =-\frac{1}{\rho^{2}} \sum_{i=1}^{2} \beta_{m i} \frac{\partial^{2} \mu_{i}}{\partial \phi^{2}}, \quad m=1,2, \\
v_{\rho} & =0, \\
v_{\phi} & =-\frac{\varepsilon k T}{2 \pi \eta Z^{2} e^{2} \rho}\left[(\bar{\zeta}+\ln \cosh \bar{\zeta}) \frac{\partial \mu_{1}}{\partial \phi}\right. \\
& \left.+(-\bar{\zeta}+\ln \cosh \bar{\zeta}) \frac{\partial \mu_{2}}{\partial \phi}\right],
\end{aligned}
$$

where the relaxation coefficients

$$
\begin{aligned}
\beta_{11}= & \frac{1}{\kappa}\left[4\left(1+\frac{3 f_{1}}{Z^{2}}\right) \exp (\bar{\zeta}) \sinh \bar{\zeta}\right. \\
& \left.-\frac{12 f_{1}}{Z^{2}}(\bar{\zeta}+\ln \cosh \bar{\zeta})\right],
\end{aligned}
$$

$$
\begin{aligned}
\beta_{12}= & -\frac{1}{\kappa}\left(\frac{12 f_{1}}{Z^{2}}\right) \ln \cosh \bar{\zeta}, \\
\beta_{21}= & -\frac{1}{\kappa}\left(\frac{12 f_{2}}{Z^{2}}\right) \ln \cosh \bar{\zeta}, \\
\beta_{22}= & \frac{1}{\kappa}\left[-4\left(1+\frac{3 f_{2}}{Z^{2}}\right) \exp (-\bar{\zeta}) \sinh \bar{\zeta}\right. \\
& \left.+\frac{12 f_{2}}{Z^{2}}(\bar{\zeta}-\ln \cosh \bar{\zeta})\right] .
\end{aligned}
$$

In the above equations, $\kappa=\left(8 \pi Z^{2} e^{2} n^{\infty} / \varepsilon k T\right)^{1 / 2}$ is the Debye screening parameter, $f_{m}=\varepsilon k^{2} T^{2} / 6 \pi \eta e^{2} D_{m}$, and $\bar{\zeta}$ was defined by Eq. [4]. The physical meaning of Eq. [10] is that the net tangential ionic fluxes along the particle surface must be balanced by the normal ionic fluxes occurring just beyond the double layer to prevent accumulation of the ionic species. The apparent slip velocity given by Eq. [11b] results from the local diffusioosmosis caused by the electrostatic interaction between the cylinder and the diffuse ions in the double layer and by the tangential gradients $\partial \mu_{m} / \rho \partial \phi$ along the cylinder surface. To obtain Eqs. [10]-[12], it was assumed that the fluid is only slightly nonuniform in the undisturbed electrolyte concentration on the length scale of $a$ and the concentration of each ionic species within the double layer is related to the electric potential by a Boltzmann distribution.

The electrochemical potentials undisturbed by the presence of the cylinder in a cell can be expressed as

$$
\mu_{m}^{\infty}=\mu_{m}^{0}+k T\left[1-(-1)^{m} \lambda\right] \ln n^{\infty},
$$

where $\lambda$ was defined by Eq. [3]. It is understood that diffusioosmosis of an electrolyte solution in a porous medium results from a linear combination of two effects: (i) chemiosmosis due to the nonuniform adsorption of counterions in the electric double layer over the charged surface, which is analogous to diffusioosmosis of a nonionic solution (27) and (ii) electroosmosis due to the macroscopic electric field generated by the concentration gradient of the electrolyte and the difference in mobilities of the cation and anion of the electrolyte, given by the second term in the brackets of Eq. [13] $(3,4,28)$. The term in Eq. [2] proportional to $\lambda$ represents the contribution from electroosmosis, while the other term is the chemiosmotic component.

At the outer (virtual) surface of the cell, the local electrochemical potential gradient of type $m$ ions is in accord with the gradient $\nabla \mu_{m}^{\infty}$. Thus $(14,25)$

$$
\rho=b: \quad \frac{\partial \mu_{m}}{\partial \rho}=k T\left[1-(-1)^{m} \lambda\right] \frac{\left(\nabla n^{\infty}\right)_{\mathrm{t}}}{n_{0}} \cos \phi, \quad m=1,2,
$$

where $n_{0}$ is the electrolyte concentration measured at the axis of the cylinder in the absence of the cylinder, and it has been assumed that $a\left(\nabla n^{\infty}\right)_{\mathrm{t}} / n_{0} \ll 1$. The solution to Eqs. [6], [10], 
and [14] is

$$
\begin{aligned}
\mu_{m}= & \mu_{m}^{0}+k T\left[1-(-1)^{m} \lambda\right] \ln n_{0} \\
& +k T \frac{\left(\nabla n^{\infty}\right)_{\mathrm{t}}}{n_{0}}\left(A_{m 1} \rho+A_{m 2} \frac{a^{2}}{\rho}\right) \cos \phi,
\end{aligned}
$$

where

$$
\begin{aligned}
& A_{m 1}=1-(-1)^{m} \lambda+\varphi A_{m 2}, \\
& A_{m 2}=\frac{1}{\Delta}\left[c_{m}^{\prime} \Delta_{1}-\Delta_{3} \varphi-(-1)^{m} \lambda\left(c_{m} \Delta_{1}-\Delta_{3} \varphi\right)\right] .
\end{aligned}
$$

In Eq. [16],

$$
\begin{aligned}
c_{1}= & \frac{1}{a^{2} \Delta_{1}}\left(a^{2}-a \beta_{11}+2 a \beta_{12}+a \beta_{22}\right. \\
& \left.+\beta_{12} \beta_{21}-\beta_{11} \beta_{22}\right), \\
c_{2}= & \frac{1}{a^{2} \Delta_{1}}\left(a^{2}+a \beta_{11}+2 a \beta_{21}-a \beta_{22}\right. \\
& \left.+\beta_{12} \beta_{21}-\beta_{11} \beta_{22}\right), \\
c_{1}^{\prime}= & c_{1}-4 \frac{\beta_{12}}{a \Delta_{1}}, \\
c_{2}^{\prime}= & c_{2}-4 \frac{\beta_{21}}{a \Delta_{1}}, \\
\Delta_{0}= & \Delta_{1}-\Delta_{2} \varphi+\Delta_{3} \varphi^{2}, \\
\Delta_{1}= & \frac{1}{a^{2}}\left(a^{2}+a \beta_{11}+a \beta_{22}-\beta_{12} \beta_{21}+\beta_{11} \beta_{22}\right), \\
\Delta_{2}= & \frac{1}{2}\left(c_{1}+c_{1}^{\prime}+c_{2}+c_{2}^{\prime}\right) \Delta_{1}, \\
\Delta_{3}= & 4-\left(\Delta_{1}+\Delta_{2}\right),
\end{aligned}
$$

and $\varphi=(a / b)^{2}$. In the limit $\kappa a \rightarrow \infty$, the effect of double-layer polarization disappears, $\beta_{11}=\beta_{12}=\beta_{21}=\beta_{22}=0, \Delta_{1}=1$, and $c_{1}=c_{2}=c_{1}^{\prime}=c_{2}^{\prime}=1$.

An alternative for the boundary condition of the electrochemical potential of type $m$ ions at the virtual surface $\rho=b$ may be taken as the distribution giving rise to the gradient $\nabla \mu_{m}^{\infty}$ in the cell when the cylinder does not exist $(16,25)$. In this case, Eq. [14] becomes

$$
\begin{aligned}
\rho=b: \quad \mu_{m}= & \mu_{m}^{0}+k T\left[1-(-1)^{m} \lambda\right] \\
& \times\left[\ln n_{0}+\frac{\left(\nabla n^{\infty}\right)_{\mathrm{t}}}{n_{0}} \rho \cos \phi\right], \quad m=1,2 .
\end{aligned}
$$

The solution of the governing equation [6] subject to the boundary conditions [10] and [21] is also given by the form of Eq. [15], but with coefficients $A_{m i}$ defined as

$$
\begin{aligned}
& A_{m 1}=1-(-1)^{m} \lambda-\varphi A_{m 2}, \\
& A_{m 2}=\frac{1}{\Delta^{\prime}}\left[c_{m}^{\prime} \Delta_{1}+\Delta_{3} \varphi-(-1)^{m} \lambda\left(c_{m} \Delta_{1}+\Delta_{3} \varphi\right)\right],
\end{aligned}
$$

where

$$
\Delta^{\prime}=\Delta_{1}+\Delta_{2} \varphi+\Delta_{3} \varphi^{2}
$$

For the particular case of $c_{1}=c_{2}=c_{1}^{\prime}=c_{2}^{\prime}=0$ and $\Delta_{1}=4$ (or $\beta_{11} / a=\beta_{22} / a=1$ and $\beta_{12} / a=\beta_{21} / a=0$ ), both Eq. [16] and Eq. [22] give $A_{11}=1+\lambda, A_{21}=1-\lambda$, and $A_{12}=A_{22}=0$, and the concentration gradient in the fluid solution resulting from Eq. [15] equals the constant imposed value everywhere.

With knowledge of the solution for the electrochemical potential distributions (so that the apparent slip velocity given by Eq. [11b] is known), we can now proceed to find the flow field in a cell. On the outer boundary of the cell, the Happel model $(9,10)$ assumes that the radial velocity relative to the bulk flow and the shear stress are zero, viz.

$$
\begin{aligned}
\rho=b: \quad v_{\rho} & =-U_{\mathrm{t}} \cos \phi \\
\tau_{\rho \phi} & =\eta\left[\rho \frac{\partial}{\partial \rho}\left(\frac{v_{\phi}}{\rho}\right)+\frac{1}{\rho} \frac{\partial v_{\rho}}{\partial \phi}\right]=0,
\end{aligned}
$$

where $U_{\mathrm{t}}$ is the transverse diffusioosmotic velocity of the bulk fluid to be determined. A solution to Eq. [7] suitable for satisfying boundary conditions on the cylindrical surfaces is (29)

$$
\Psi=\left(C \rho^{-1}+D \rho \ln \frac{\rho}{a}+E \rho+F \rho^{3}\right) \sin \phi,
$$

where the constants $C, D, E$, and $F$ are to be determined from Eqs. [11] and [24] using Eqs. [9] and [15]. The procedure is straightforward, with the result

$$
\begin{aligned}
& C=a^{2} \omega\left[U_{\mathrm{t}}+V A \ln \varphi\right], \\
& D=2 \omega\left[U_{\mathrm{t}}\left(1+\varphi^{2}\right)-V A\left(1-\varphi^{2}\right)\right], \\
& E=-\omega\left(1-\varphi^{2}\right)\left[U_{\mathrm{t}}+V A \ln \varphi\right], \\
& F=-a^{-2} \omega \varphi^{2}\left[U_{\mathrm{t}}+V A \ln \varphi\right],
\end{aligned}
$$

where $V$ is a characteristic velocity of the fluid defined by

$$
\begin{aligned}
V= & \frac{\varepsilon}{4 \pi \eta}\left(\frac{k T}{Z e}\right)^{2} \frac{\left(\nabla n^{\infty}\right)_{\mathrm{t}}}{n_{0}}, \\
\omega= & \left(1-\varphi^{2}+\ln \varphi+\varphi^{2} \ln \varphi\right)^{-1}, \\
A= & (\bar{\zeta}+\ln \cosh \bar{\zeta})\left(A_{11}+A_{12}\right) \\
& +(-\bar{\zeta}+\ln \cosh \bar{\zeta})\left(A_{21}+A_{22}\right),
\end{aligned}
$$

and $A_{11}, A_{12}, A_{21}$, and $A_{22}$ are given by Eq. [16] or [22]. With this solution, the components of the fluid velocity can be calculated by using Eq. [9].

The drag force (in the $x$ direction) exerted by the fluid on the cylinder per unit length is (29)

$$
F_{\mathrm{d}}=4 \pi \eta D
$$


At the steady state, this net force must vanish, viz. $D=0$. With this constraint, Eq. [26b] yields the transverse diffusioosmotic velocity of the bulk fluid,

$$
U_{\mathrm{t}}=V A\left(1-\varphi^{2}\right)\left(1+\varphi^{2}\right)^{-1} .
$$

If the Kuwabara model (11) for the boundary conditions of the fluid flow at the virtual surface of the cell, which assumes that the radial velocity relative to the bulk flow and the vorticity are zero, is used, Eq. [24b] is replaced by

$$
\rho=b: \quad(\nabla \times \mathbf{v})_{z}=\frac{\partial v_{\phi}}{\partial \rho}+\frac{v_{\phi}}{\rho}-\frac{1}{\rho} \frac{\partial v_{\rho}}{\partial \phi}=0
$$

With this change, the stream function $\Psi$ can still be expressed in the form of Eq. [25], and the coefficients $C, D, E$, and $F$ should be determined by boundary conditions [11], [24a], and [32]. The result is

$$
\begin{aligned}
& C=a^{2} \omega^{\prime}\left[U_{\mathrm{t}}(2-\varphi)+V A(1-\varphi+2 \ln \varphi)\right], \\
& D=4 \omega^{\prime}\left[U_{\mathrm{t}}-V A(1-\varphi)\right], \\
& E=-\omega^{\prime}\left[2 U_{\mathrm{t}}(1-\varphi)+V A\left(1-\varphi^{2}+2 \ln \varphi\right)\right], \\
& F=-a^{-2} \omega^{\prime} \varphi\left[U_{\mathrm{t}}-V A(1-\varphi)\right],
\end{aligned}
$$

where

$$
\omega^{\prime}=\left(3+2 \ln \varphi-4 \varphi+\varphi^{2}\right)^{-1} .
$$

The fact that there is no drag force exerted on the cylinder requires $D=0$, and Eq. [33b] gives the bulk transverse diffusioosmotic velocity as

$$
U_{\mathrm{t}}=V A(1-\varphi)
$$

For any combination of $\zeta e / k T, \kappa a, Z, f_{1}, f_{2}$, and $\varphi$, the Happel model always results in a slightly higher value of $U_{\mathrm{t}}$ than the Kuwabara model does (by a factor $\left.(1+\varphi)\left(1+\varphi^{2}\right)^{-1}\right)$, which occurs because the zero-vorticity boundary condition yields a larger energy dissipation in the cell than that due to the drag on the cylinder alone. However, the Happel model has a significant advantage in that it does not require an exchange of mechanical energy between the cell and the environment (29).

As to the diffusioosmosis generated by the longitudinal component $\left(\nabla n^{\infty}\right)_{1}$ of the electrolyte gradient, there is no polarization of the thin double layers or disturbance in the fluid velocity and ionic electrochemical potential fields caused by the curvature of the cylinders like the above analysis for the transverse motion. The longitudinal diffusioosmotic velocity of the electrolyte solution is given by Eq. [2], and the bulk fluid velocity in the negative $z$ direction through the ordered array of cylinders can be expressed as

$$
U_{1}=\frac{\varepsilon \zeta}{4 \pi \eta}\left(\lambda+\bar{\zeta}^{-1} \ln \cosh \bar{\zeta}\right) \frac{k T}{Z e}\left(\nabla \ln n^{\infty}\right)_{1} .
$$

The total diffusioosmotic velocity of the bulk fluid caused by an arbitrarily oriented electrolyte gradient is the vectorial sum of the transversal and longitudinal contributions,

$$
\mathbf{U}=-\left(U_{\mathrm{t}} \mathbf{e}_{x}+U_{1} \mathbf{e}_{z}\right) .
$$

Thus, in general, the direction of the bulk diffusioosmotic flow will not be parallel to the prescribed electrolyte gradient.

\section{RESULTS OF DIFFUSIOOSMOSIS}

Due to the differences in the boundary conditions for the electrochemical potential and fluid velocity distributions at the virtual surface $\rho=b$ of a unit cell, four cases of the cell model can be defined. Case I: the Happel model with a specified electrochemical potential gradient at the virtual surface (the boundary conditions at $\rho=b$ are described by Eqs. [14] and [24]); Case II: the Happel model with a specified electrochemical potential profile at the virtual surface (the boundary conditions at $\rho=b$ are described by Eqs. [21] and [24]); Case III: the Kuwabara model with a specified electrochemical potential gradient at the virtual surface (the boundary conditions at $\rho=b$ are described by Eqs. [14], [24a], and [32]); Case IV: the Kuwabara model with a specified electrochemical potential profile at the virtual surface (the boundary conditions at $\rho=b$ are described by Eqs. [21], [24a], and [32]). The analytical solutions of the ionic electrochemical potentials and the fluid flow in the unit cell and the diffusioosmotic velocity of the bulk fluid have been obtained in the previous section for all of the four cases.

In Case I, the transverse diffusioosmotic velocity is given by Eq. [31] with coefficient $A$ defined by Eqs. [29] and [16]. This velocity can be expressed as

$$
U_{\mathrm{t}}=U_{\mathrm{t}}^{(0)}\left[1+\frac{1}{\Delta}\left(h \varphi-\Delta_{3} \varphi^{2}\right)\right]\left(1-\varphi^{2}\right)\left(1+\varphi^{2}\right)^{-1},
$$

where

$$
U_{\mathrm{t}}^{(0)}=\frac{Z e \zeta}{4 k T} g V,
$$

which is the transverse diffusioosmotic velocity of the bulk fluid in the limit $\varphi=0$; the parameters $g$ and $h$ are

$$
\begin{aligned}
g= & c_{1}^{\prime}-c_{2}^{\prime}+\lambda\left(2+c_{1}+c_{2}\right) \\
& +\left[2+c_{1}^{\prime}+c_{2}^{\prime}+\lambda\left(c_{1}-c_{2}\right)\right] \bar{\zeta}^{-1} \ln \cosh \bar{\zeta} \\
h= & \frac{1}{g}\left\{\left(c_{1}^{\prime}-c_{2}^{\prime}\right)\left(\Delta_{1}+\Delta_{2}\right)+\lambda\left[\left(c_{1}+c_{2}\right)\left(\Delta_{1}+\Delta_{2}\right)-2 \Delta_{3}\right]\right. \\
& +\left[\left(c_{1}^{\prime}+c_{2}^{\prime}\right)\left(\Delta_{1}+\Delta_{2}\right)-2 \Delta_{3}\right. \\
& \left.\left.+\lambda\left(c_{1}-c_{2}\right)\left(\Delta_{1}+\Delta_{2}\right)\right] \bar{\zeta}^{-1} \ln \cosh \bar{\zeta}\right\} .
\end{aligned}
$$


Equation [39] with $V$ and $g$ given by Eqs. [27] and [40] can also be used to express the corresponding diffusiophoretic velocity of an isolated charged circular cylinder in the direction normal to its axis (27). Note that, in the limit $\kappa a \rightarrow \infty$, Eq. [39] reduces to Eq. [2].

In Case II, the transverse diffusioosmotic velocity of the bulk fluid within the array of charged cylinders can be evaluated by Eq. [31] with $A$ given by Eqs. [29] and [22], and its expression parallel to Eq. [38] for Case I is

$$
U_{\mathrm{t}}=U_{\mathrm{t}}^{(0)}\left[1-\frac{1}{\Delta^{\prime}}\left(h \varphi+\Delta_{3} \varphi^{2}\right)\right]\left(1-\varphi^{2}\right)\left(1+\varphi^{2}\right)^{-1} .
$$

In Case III, the transverse diffusioosmotic velocity of the bulk fluid can be obtained by Eqs. [35], [29], and [16], with the result

$$
U_{\mathrm{t}}=U_{\mathrm{t}}^{(0)}\left[1+\frac{1}{\Delta}\left(h \varphi-\Delta_{3} \varphi^{2}\right)\right](1-\varphi) .
$$

In Case IV, the bulk transverse fluid velocity is given by Eq. [35] with $A$ defined by Eqs. [29] and [22], and it can be expressed as

$$
U_{\mathrm{t}}=U_{\mathrm{t}}^{(0)}\left[1-\frac{1}{\Delta^{\prime}}\left(h \varphi+\Delta_{3} \varphi^{2}\right)\right](1-\varphi) .
$$

It can be found from Eqs. [38]-[44] that the transverse diffusioosmotic velocity of the electrolyte solution in the fiber matrix predicted by the cell model is quite sensitive to the boundary conditions specified at the virtual surface of the cell. The boundary condition for the electrolyte concentration or ionic electrochemical potentials at the virtual surface $\rho=b$ determines the

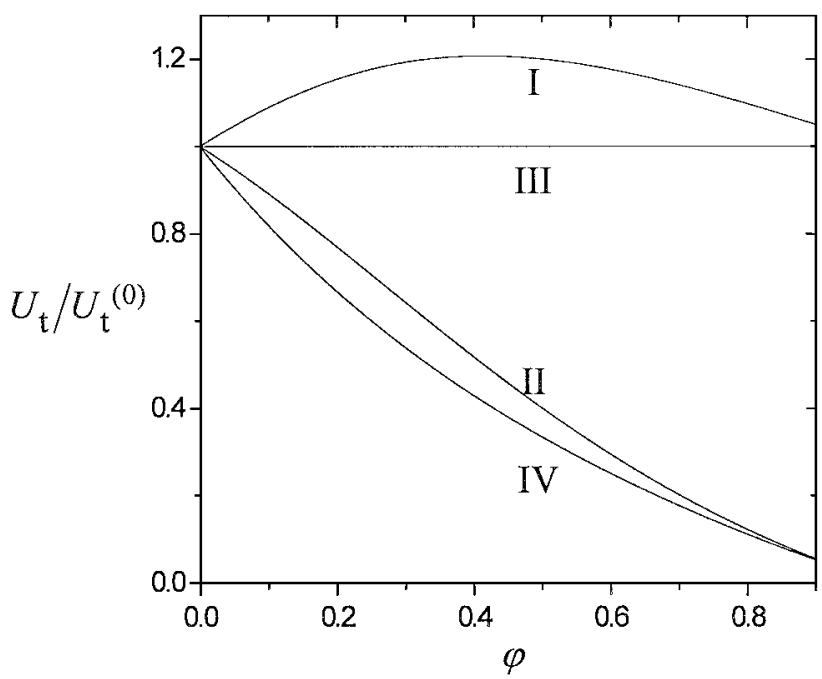

FIG. 2. Plots of the normalized transverse diffusioosmotic velocity of an electrolyte solution in an ordered array of identical cylinders versus $\varphi$ in the limit $\kappa a \rightarrow \infty$. The curves with labels I, II, III, and IV represent calculations from Eqs. [38], [42], [43], and [44], respectively.
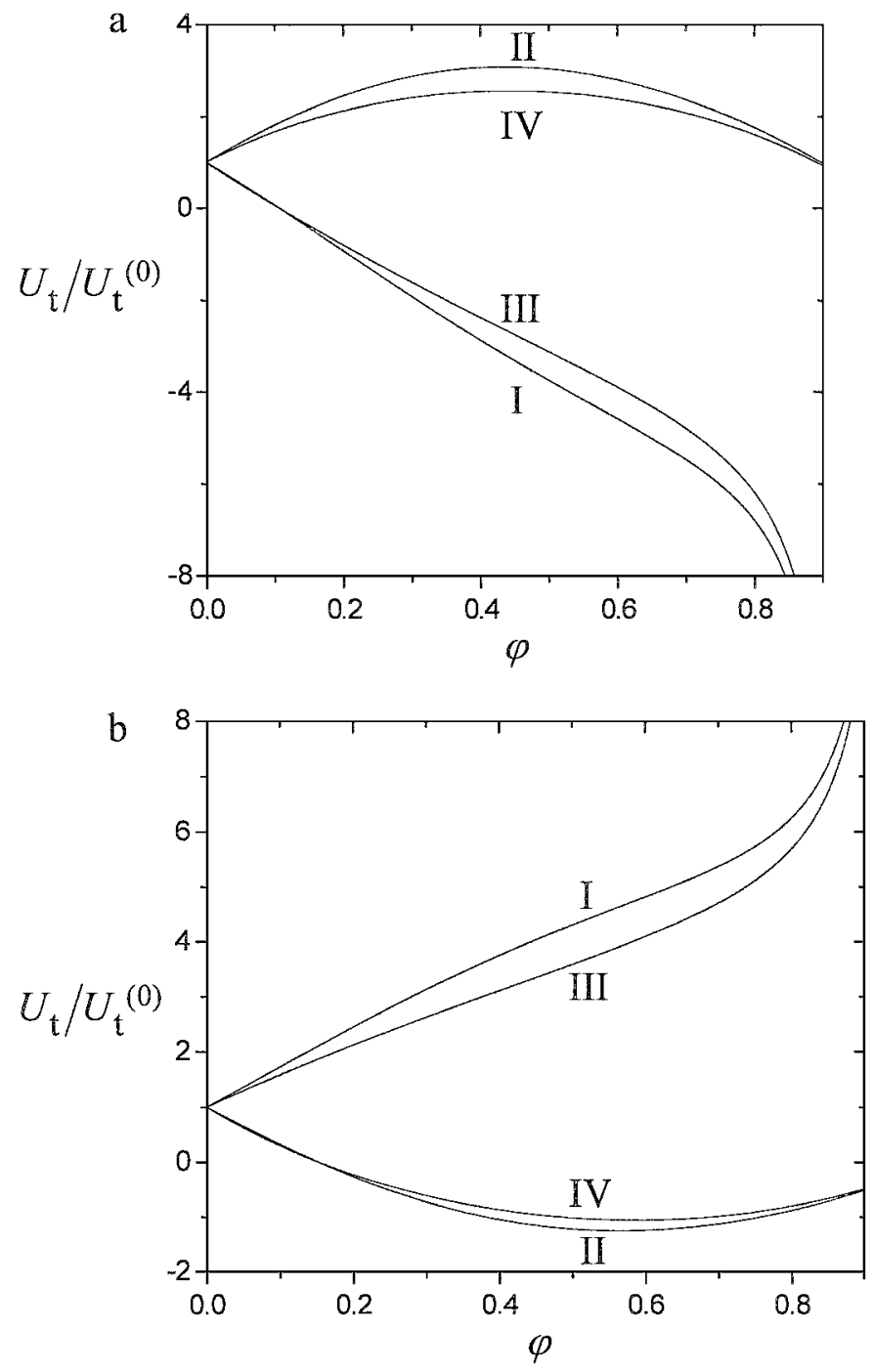

FIG. 3. Plots of the normalized transverse diffusioosmotic velocity of an electrolyte solution in an ordered array of identical cylinders versus $\varphi$ with $Z=1, f_{1}=0.2$, $\zeta e / k T=8$, and $\kappa a=50$ : (a) $\lambda=0$; (b) $\lambda=-0.2$. The curves with labels I, II, III, and IV represent calculations from Eqs. [38], [42], [43], and [44], respectively.

dependence of the normalized diffusioosmotic velocity $U_{\mathrm{t}} / U_{\mathrm{t}}^{(0)}$ as a function of the properties of the cylinder and the electrolyte solution (such as $\zeta e / k T$ and $\kappa a$ ), while the boundary condition for the fluid velocity at $\rho=b$ controls the connection of $U_{\mathrm{t}} / U_{\mathrm{t}}^{(0)}$ with the remainder part.

The normalized transverse diffusioosmotic velocity of the bulk fluid within the array of charged cylinders, $U_{\mathrm{t}} / U_{\mathrm{t}}^{(0)}$, as calculated from Eqs. [38]-[44] for the four cases of the cell model, is plotted versus the volume fraction of the cylinders, $\varphi$, in Figs. 2 and 3 for various values of the parameters $Z, \lambda, \zeta e / k T$, and $\kappa a$. In all cases, $U_{\mathrm{t}} / U_{\mathrm{t}}^{(0)}=1$ in the limit $\varphi=0$. The calculations are presented up to $\varphi=0.9$, which corresponds to the maximum attainable volume fraction for a swarm of identical cylinders (ordered triangularly, 30). 
In Fig. 2, the normalized diffusioosmotic velocity $U_{\mathrm{t}} / U_{\mathrm{t}}^{(0)}$ of the fluid in the fiber matrix is plotted as a function of $\varphi$ in the limit of $\kappa a \rightarrow \infty$. It is clearly shown that the Kuwabara model predicts a somewhat smaller diffusioosmotic mobility than the Happel model does under otherwise the same conditions. In this limiting situation, $U_{\mathrm{t}} / U_{\mathrm{t}}^{(0)}$ is greater than unity at all values of $\varphi$ for Case I, is a monotonically decreasing function of $\varphi$ for Cases II and IV, and is independent of $\varphi$ for Case III.

The results of $U_{\mathrm{t}} / U_{\mathrm{t}}^{(0)}$ as a function of $\varphi$ for the electrolyte solution within the array of charged cylinders with a finite value of $\kappa a$ are plotted in Fig. 3 for the four cases of the cell model. The situations associated with Fig. $3 \mathrm{a}\left(Z=1, f_{1}=f_{2}=0.2\right)$ and $3 \mathrm{~b}\left(Z=1, f_{1}=0.2, \lambda=-0.2\right)$ are close to those of diffusioosmosis of the aqueous solutions of $\mathrm{KCl}$ and $\mathrm{NaCl}$, respectively. It can be seen in Figs. 2 and 3 that the four cases of the cell model lead to quite different results of the transverse diffusioosmotic velocity of the fluid. Note that the bulk fluid can reverse its direction of flow with a decrease in the porosity (an increase in $\varphi$ ) of the fiber matrix.

The unit cell models with various boundary conditions at the virtual surface of the cell have also been used in the literature to study the electrophoresis (14-17) and diffusiophoresis (25) of suspensions of charged spheres with thin double layers. The results of those studies indicate that the tendency of the dependence of the normalized particle mobility on the volume fraction of the particles in Cases I and III is not correct in comparison with the corresponding ensemble-averaged results obtained by using the concept of statistical mechanics. Thus, the boundary condition represented by Eq. [14] might not be as accurate as that represented by Eq. [21], probably due to the fact that the angular component of the gradients of the electrochemical potential energies at the virtual surface of the cell is not specified in Eq. [14]. It was also shown that the electrophoretic and diffusiophoretic velocities predicted by Case IV of the cell model agree quite well with those obtained from the statistical model (31) and from an experimental study (32) for dilute suspensions of particles. A possible reason for this outcome might be the fact that the Kuwabara boundary condition of zero vorticity is consistent with the irrotational-flow environment generated by an electrophoretic or diffusiophoretic particle with a thin double layer. Note that, however, except for the case with $\kappa a \rightarrow \infty$, the flow caused by two or more identical diffusiophoretic or electrophoretic spheres is not irrotational (31).

In Fig. $4, U_{\mathrm{t}} / U_{\mathrm{t}}^{(0)}$ of the electrolyte solution within the array of charged cylinders is plotted as a function of $\varphi$ for Case IV with $Z$ and $\zeta e / k T$ as parameters. It can be seen that the normalized transverse diffusioosmotic velocity of the bulk fluid can be increased or decreased by the increase of $\varphi$, depending on the values of the relevant factors. Again, Fig. 4 illustrates that the bulk fluid can reverse its direction of flow with the variation of $\varphi$, keeping these factors unchanged. The results in Fig. 4 reveal the drastic effect of ion valence on the diffusioosmotic velocity, in particular for large normalized zeta potential. The physical
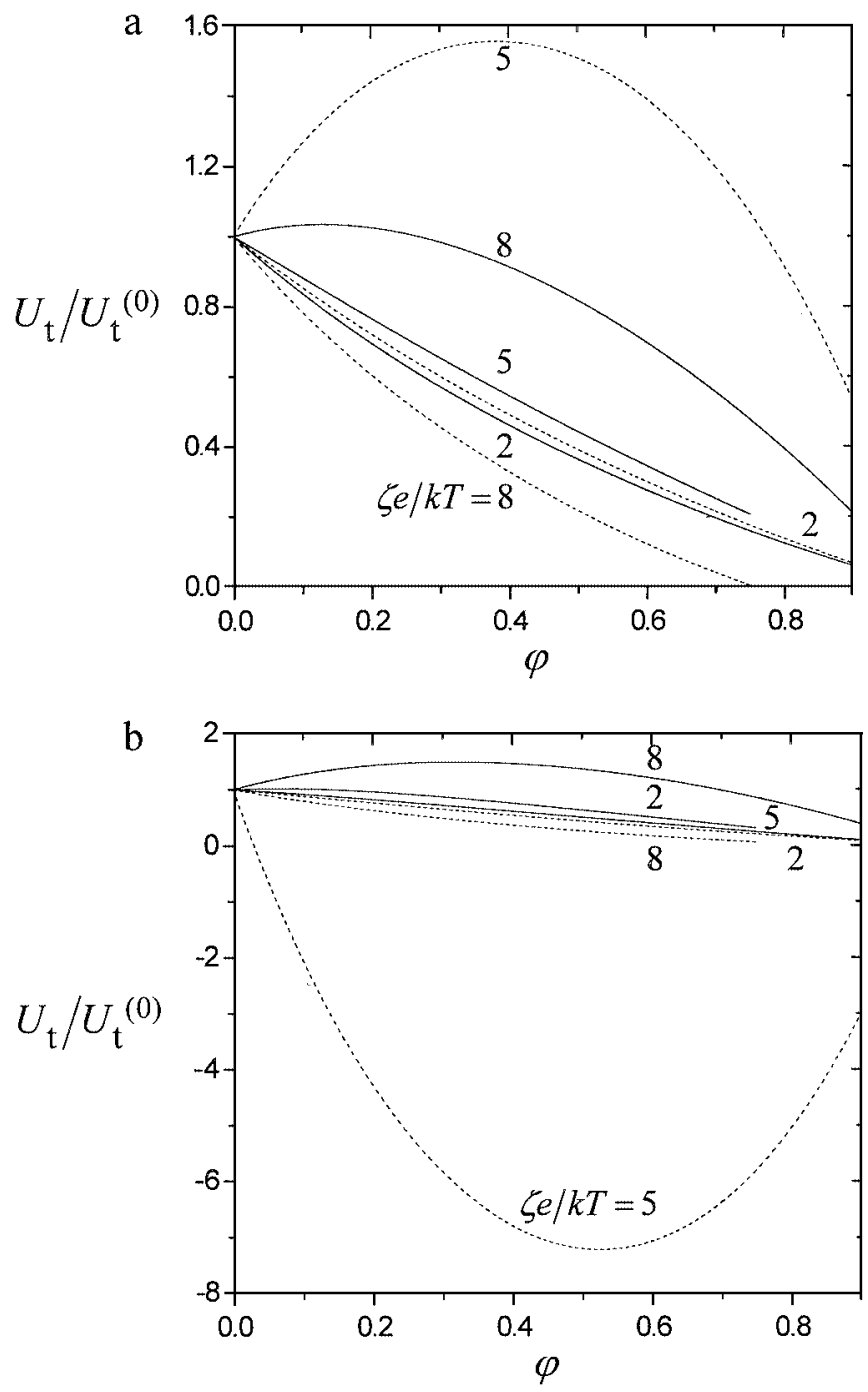

FIG. 4. Plots of the normalized transverse diffusioosmotic velocity of an electrolyte solution in an ordered array of identical cylinders versus $\varphi$ for Case IV with $f_{1}=0.2$ and $\kappa a=100$ : (a) $\lambda=0$; (b) $\lambda=-0.2$. Solid curves represent the case $Z=1$ and dashed curves denote the case $Z=2$.

explanation for it will be given with the discussion on the results in Fig. 5.

The normalized diffusioosmotic velocity $U_{\mathrm{t}} / U_{\mathrm{t}}^{(0)}$ of the electrolyte solution within the array of charged cylinders is plotted versus their dimensionless zeta potential at different values of $\kappa a$ and $Z$ in Fig. 5 for Case IV when $\varphi$ is kept constant. In Fig. 5a for the case of $\lambda=0$, only the results at positive zeta potentials are displayed since the induced macroscopic electric field vanishes and the bulk fluid velocity, which is due to the chemiosmotic effect only, will be an even function of the zeta potential. When the value of $Z e \zeta / k T$ is small (say, <6), $U_{\mathrm{t}} / U_{\mathrm{t}}^{(0)}$ is a monotonically increasing function of $Z e \zeta / k T$ for a finite value of $\kappa a$. Also, this diffusioosmotic mobility is larger with smaller $\kappa a$. However, when the value of $Z e \zeta / k T$ is increased, a maximum and a minimum of the normalized bulk fluid velocity would appear. 
As $Z$ increases or $\kappa a$ decreases, the extremes occur at smaller zeta potentials. Note that the abrupt variation of the normalized diffusioosmotic velocity near these extremes is due to the fact that the direction of the velocity $U_{\mathrm{t}}^{(0)}$ given by Eq. [39] reverses and its magnitude is small over there (27), while the velocity $U_{\mathrm{t}}$ does not reverse synchronously. In Fig. $5 b$ for the case of $\lambda \neq 0$, both the chemiosmotic and electroosmotic effects contribute to the fluid motion and the net diffusioosmotic velocity is neither an even nor an odd function of $\zeta$. Again, $U_{\mathrm{t}} / U_{\mathrm{t}}^{(0)}$ is not a monotonic
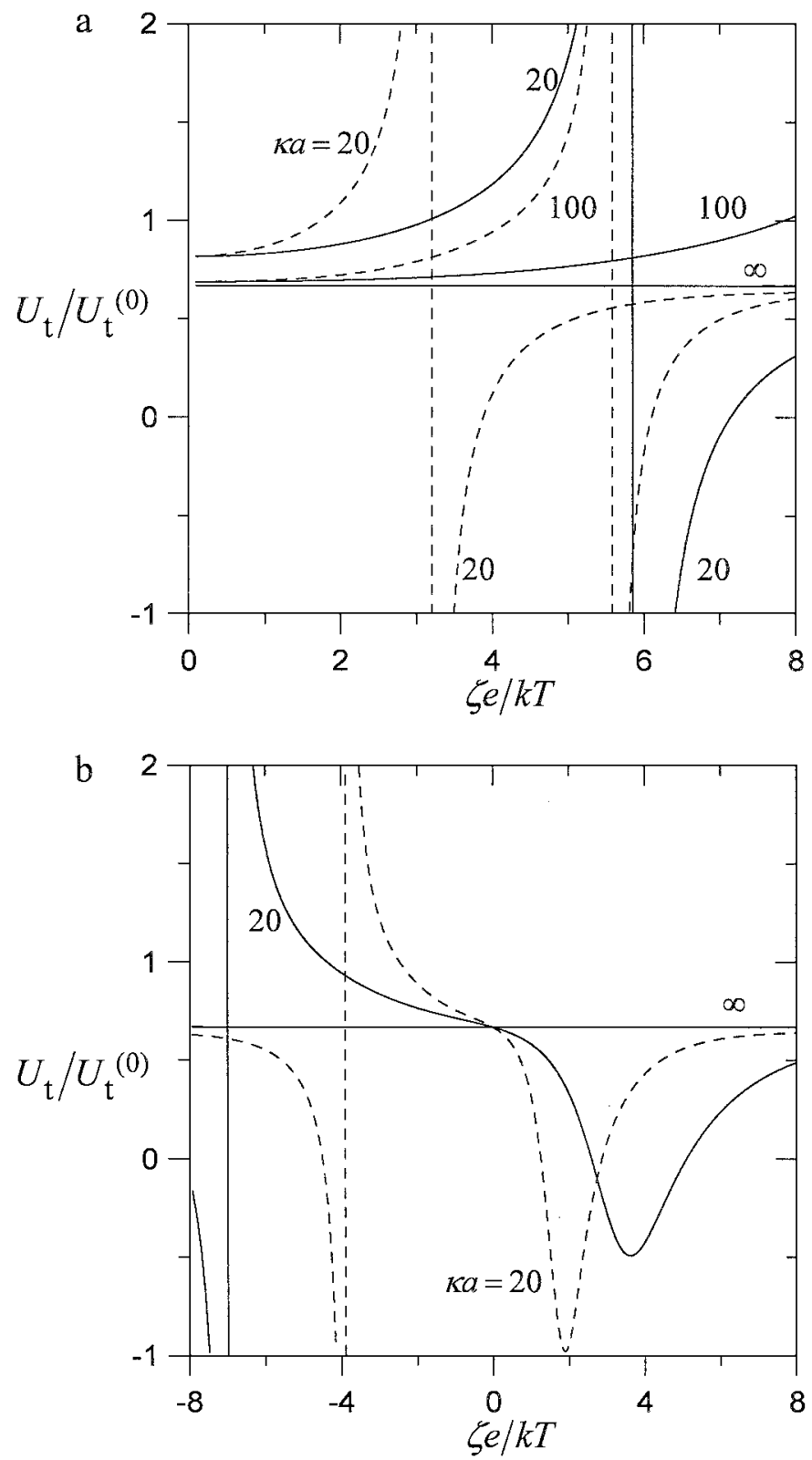

FIG. 5. Plots of the normalized transverse diffusioosmotic velocity of an electrolyte solution in an ordered array of identical cylinders versus the dimensionless zeta potential $\zeta e / k T$ for Case IV with $f_{1}=0.2$ and $\varphi=0.2:$ (a) $\lambda=0$; (b) $\lambda=-0.2$. Solid curves represent the case $Z=1$ and dashed curves denote the case $Z=2$.

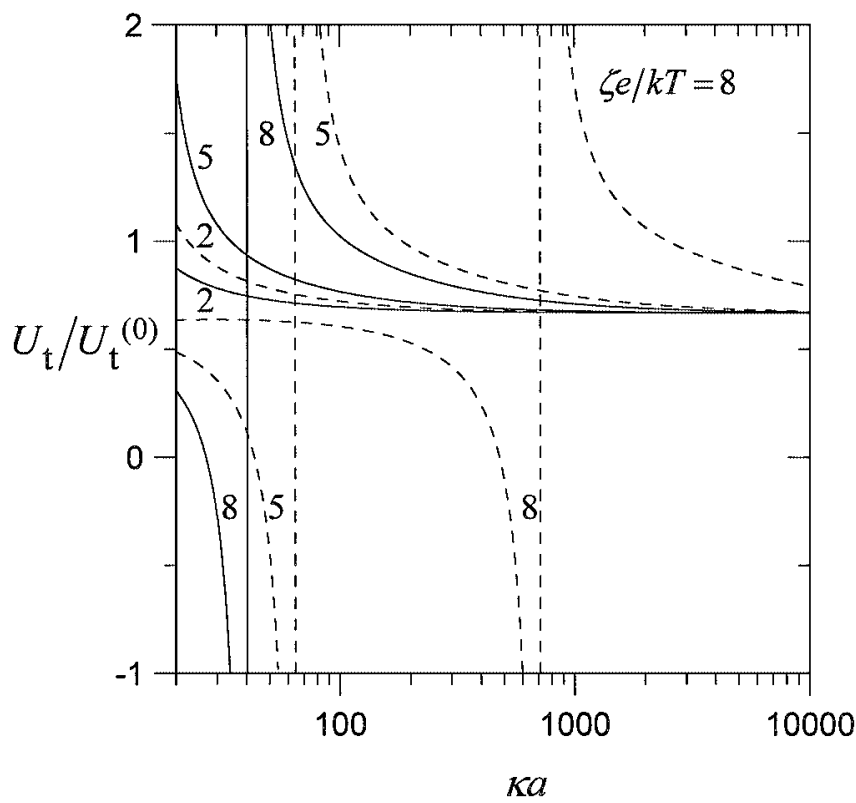

FIG. 6. Plots of the normalized transverse diffusioosmotic velocity of an electrolyte solution in an ordered array of identical cylinders versus $\kappa a$ for Case IV with $f_{1}=f_{2}=0.2$ and $\varphi=0.2$. Solid curves represent the case $Z=1$ and dashed curves denote the case $Z=2$.

function of $\zeta e / k T$. In general, no simple rule could appropriately describe the flow behavior. Whether the diffusioosmotic mobility of the fluid is increased or decreased depends on the combination of $\zeta e / k T, \kappa a, Z, f_{1}$, and $f_{2}$.

In Fig. $6, U_{\mathrm{t}} / U_{\mathrm{t}}^{(0)}$ of the electrolyte solution is plotted versus $\kappa a$ in the range from 20 to 10,000 for Case IV with $Z$ and $\zeta e / k T$ as parameters. For the case $Z=1$, the magnitude of $U_{\mathrm{t}} / U_{\mathrm{t}}^{(0)}$ in general decreases steadily as $\kappa a$ becomes large gradually. However, when the value of $Z e \zeta / k T$ gets large (say, $>6$ ), there can be a minimum and a maximum of the normalized diffusioosmotic velocity occurring at some value of $\kappa a$ for a given value of $\zeta e / k T$. If the cylinders are charged more highly (with greater magnitude in zeta potential) or the counterions have a larger absolute value of valence, the locations of these extremes will shift toward large $\kappa a$; that means larger values of $\kappa a$ are required to make the assumption of $\kappa a \rightarrow \infty$ valid. Although only the situation of $\lambda=0$ is displayed in Fig. 6 , the plot of $U_{\mathrm{t}} / U_{\mathrm{t}}^{(0)}$ versus $\kappa a$ for cases with $\lambda \neq 0$ will indicate a similar outcome.

\section{ELECTROOSMOSIS}

Considered in this section is the steady electroosmotic flow of a solution of a symmetrically charged electrolyte in the fibrous porous medium constructed by an array of charged cylinders subjected to a uniform electric field $\mathbf{E}^{\infty}$. The bulk concentration $n^{\infty}$ of the symmetric electrolyte beyond the electric double layers is constant now. The thickness of the double layers is assumed to be much smaller than the radius of the cylinders and the 
surface-to-surface distance between the neighboring cylinders, but the polarization effect in the thin diffuse layers is allowed. Like the analysis in Section 2, we can decompose $\mathbf{E}^{\infty}$ into the transversal and longitudinal components, $E_{\mathrm{t}}^{\infty} \mathbf{e}_{x}$ and $E_{1}^{\infty} \mathbf{e}_{z}$, and discuss the electroosmosis due to each separately.

First, we examine the fluid motion due to the transverse component $E_{\mathrm{t}}^{\infty}$ of the applied electric field. The bulk electroosmotic velocity caused by this component can be expressed by $-U_{\mathrm{t}} \mathbf{e}_{x}$. Again, the unit cell model shown in Fig. 1 is employed. Outside the double layer in each cell, the electrochemical potentials of the ions satisfy the Laplace equation [6] and the boundary condition of Eq. [10] at the cylinder surface, but their undisturbed values are given by

$$
\mu_{m}^{\infty}=\mu_{m}^{0}+k T \ln n^{\infty}-(-1)^{m} Z e E_{\mathrm{t}}^{\infty} \rho \cos \phi,
$$

in replacement of Eq. [13]. If a boundary condition similar to Eq. [14] is used at the virtual surface of the cell, one has

$$
\rho=b: \quad \frac{\partial \mu_{m}}{\partial \rho}=-(-1)^{m} Z e E_{\mathrm{t}}^{\infty} \cos \phi, \quad m=1,2,
$$

where $m$ equal to 1 and 2 refers to the anion and cation, respectively, of the symmetric electrolyte of valence $Z$. The solution to Eqs. [6], [10], and [46] is

$$
\mu_{m}=\mu_{m}^{0}+k T \ln n^{\infty}+Z e E_{\mathrm{t}}^{\infty}\left(A_{m 1} \rho+A_{m 2} \frac{a^{2}}{\rho}\right) \cos \phi,
$$

where, instead of Eq. [16],

$$
\begin{aligned}
& A_{m 1}=-(-1)^{m}+\varphi A_{m 2}, \\
& A_{m 2}=-(-1)^{m} \frac{1}{\Delta}\left(c_{m} \Delta_{1}-\Delta_{3} \varphi\right) .
\end{aligned}
$$

If a boundary condition analogous to Eq. [21],

$$
\rho=b: \quad \mu_{m}=\mu_{m}^{\infty}, \quad m=1,2,
$$

is used, the solution of the electrochemical potentials is also given by Eq. [47], but now the coefficients $A_{m i}$ are

$$
\begin{aligned}
& A_{m 1}=-(-1)^{m}-\varphi A_{m 2}, \\
& A_{m 2}=-(-1)^{m} \frac{1}{\Delta^{\prime}}\left(c_{m} \Delta_{1}+\Delta_{3} \varphi\right) .
\end{aligned}
$$

The governing equation, boundary conditions, and solution for the fluid flow field and the expression for the transverse electroosmotic velocity $U_{\mathrm{t}}$ of the bulk fluid have the same forms as those given in Section 2, except that the characteristic velocity of the fluid given by Eq. [27] should be changed into

$$
V=\frac{\varepsilon k T E_{\mathrm{t}}^{\infty}}{4 \pi \eta Z e} .
$$

The final results for the transverse electroosmotic velocity of the bulk fluid for all of the four cases of the cell model also have the same forms as those presented in Section 3, except that $g$ and $h$ in Eqs. [40] and [41] are replaced by

$$
\begin{aligned}
g= & 2+c_{1}+c_{2}+\left(c_{1}-c_{2}\right) \bar{\zeta}^{-1} \ln \cosh \bar{\zeta}, \\
h= & \frac{1}{g}\left[\left(c_{1}+c_{2}\right)\left(\Delta_{1}+\Delta_{2}\right)-2 \Delta_{3}\right. \\
& \left.+\left(c_{1}-c_{2}\right)\left(\Delta_{1}+\Delta_{2}\right) \bar{\zeta}^{-1} \ln \cosh \bar{\zeta}\right] .
\end{aligned}
$$

Now, $U_{\mathrm{t}}^{(0)}$ given by Eq. [39] with $V$ and $g$ given by Eqs. [51] and [52] is the transverse electroosmotic velocity of the bulk fluid in the limit $\varphi=0$, which is also the electrophoretic velocity of an isolated charged cylindrical particle with a thin but polarized double layer caused by the transversely applied electric field (27). In the limit $\kappa a \rightarrow \infty, U_{\mathrm{t}}^{(0)}$ reduces to the Helmholtz result given by Eq. [1].

For the electroosmosis driven by the longitudinal component $E_{1}^{\infty}$ of the imposed electric field, no polarization of the diffuse ions or disturbance in the fluid velocity and electrochemical potential fields arises. The longitudinal electroosmotic velocity of the electrolyte solution is given by the Helmholtz equation and the bulk fluid velocity in the negative $z$ direction can be written as

$$
U_{1}=\frac{\varepsilon \zeta}{4 \pi \eta} E_{1}^{\infty}
$$

Similarly, the overall electroosmotic velocity of the bulk fluid caused by an arbitrary electric field can also be determined by Eq. [37].

In the limiting situation given by $\kappa a \rightarrow \infty$, the dependence of the normalized electroosmotic velocity $U_{\mathrm{t}} / U_{\mathrm{t}}^{(0)}$ of the electrolyte solution within the array of charged cylinders on the cylinder volume fraction $\varphi$ is the same as that for the diffusioosmosis, shown in Fig. 2. The results of $U_{\mathrm{t}} / U_{\mathrm{t}}^{(0)}$ for the fluid in the fiber matrix with a finite value of $\kappa a$ are plotted in Fig. 7 for the four cases of the cell model. Similar to the corresponding situation of diffusioosmosis presented in the previous section, the four cases of the cell model yield quite different results for the electroosmotic mobility of the fluid. Note that, in the situation of electroosmosis, $U_{\mathrm{t}} / U_{\mathrm{t}}^{(0)}$ is always positive and its values obtained from Cases III and IV are smaller than those obtained from Cases I and II, respectively.

The normalized electroosmotic velocity $U_{\mathrm{t}} / U_{\mathrm{t}}^{(0)}$ of the electrolyte solution within the array of charged cylinders is plotted versus $\zeta e / k T$ at different values of $\kappa a$ and $Z$ in Fig. 8a for Case IV with a constant $\varphi$. When the value of $Z e \zeta / k T$ is small (say, $<6), U_{\mathrm{t}} / U_{\mathrm{t}}^{(0)}$ increases monotonically with an increase in $Z e \zeta / k T$ or a decrease in $\kappa a$. However, when the value of 
$Z e \zeta / k T$ is increased, a maximum of the normalized electroosmotic mobility would appear. As $Z$ increases or $\kappa a$ decreases, the maximum occurs at a smaller zeta potential.

In Fig. 8b, the results of $U_{\mathrm{t}} / U_{\mathrm{t}}^{(0)}$ for a given value of $\varphi$ are plotted versus $\kappa a$ for Case IV with $\mathrm{Z}$ and $\zeta e / k T$ as parameters. For the case $Z=1, U_{\mathrm{t}} / U_{\mathrm{t}}^{(0)}$ in general decreases as $\kappa a$ increases. However, when the value of $Z e \zeta / k T$ is large (say, $>6$ ), there can be a maximum of the normalized electroosmotic mobility occurring at some $\kappa a$ for a fixed value of $\zeta e / k T$. If the value of $Z e \zeta / k T$ is increased, the locations of these maximal values will shift toward larger $\kappa a$.
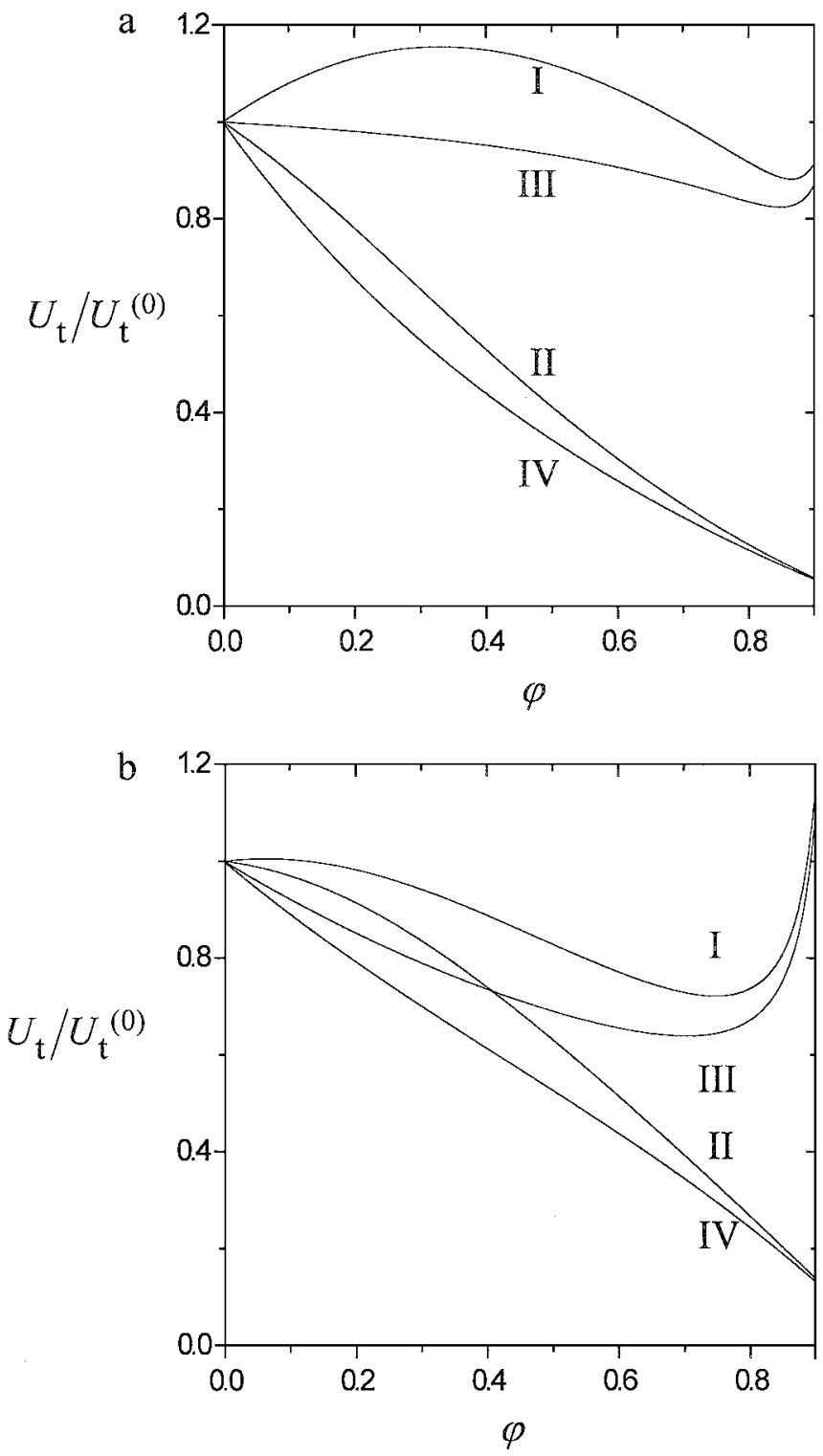

FIG. 7. Plots of the normalized transverse electroosmotic velocity of an electrolyte solution in an ordered array of identical cylinders versus $\varphi$ with $Z=$ $1, f_{1}=f_{2}=0.2$, and $\kappa a=50$ : (a) $\zeta e / k T=2$; (b) $\zeta e / k T=8$. The curves with labels I, II, III, and IV represent calculations from Eqs. [38], [42], [43], and [44], respectively.
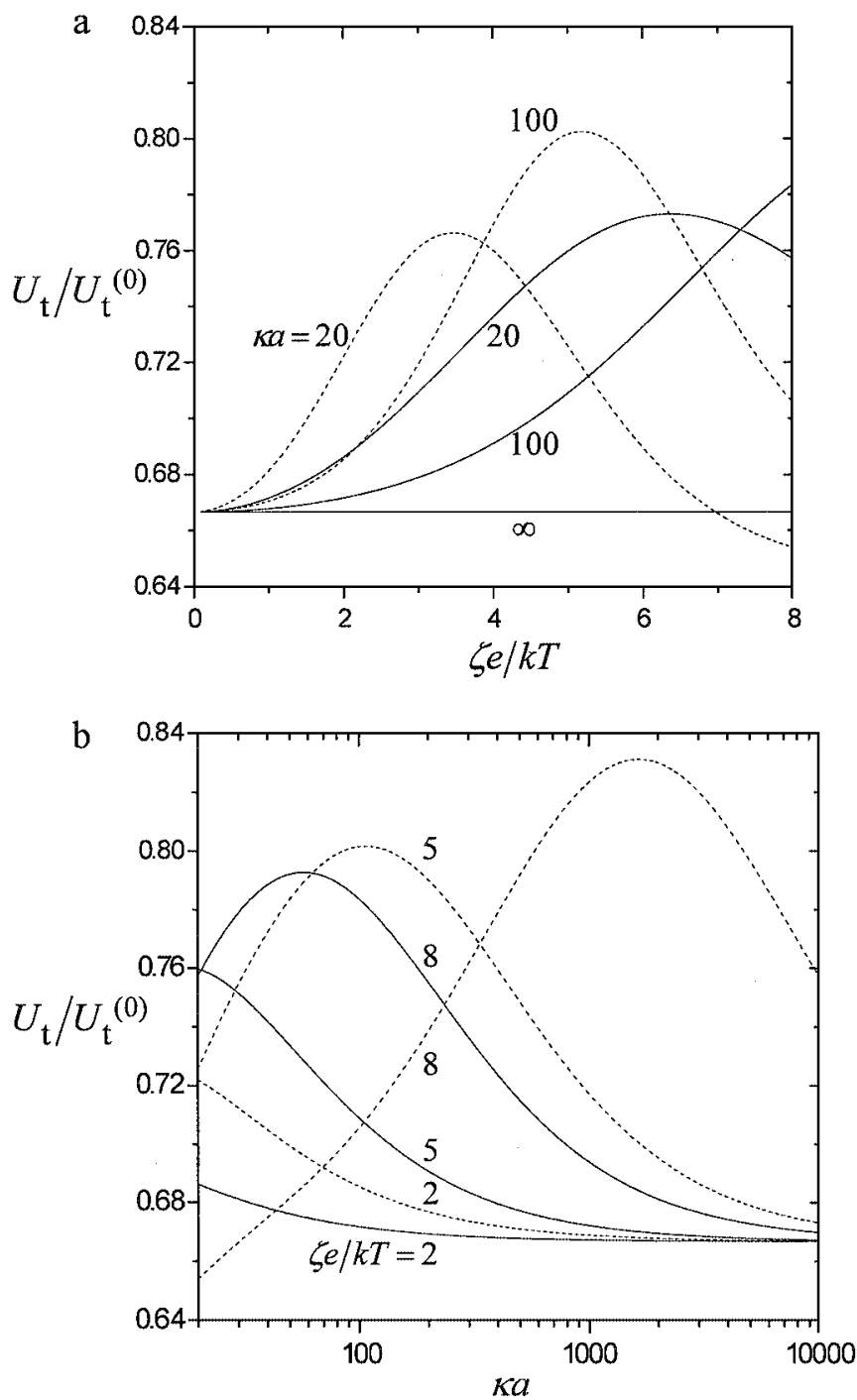

FIG. 8. Plots of the normalized transverse electroosmotic velocity of an electrolyte solution in an ordered array of identical cylinders for Case IV with $f_{1}=f_{2}=0.2$ and $\varphi=0.2$ : (a) $U_{\mathrm{t}} / U_{\mathrm{t}}^{(0)}$ versus $\zeta e / k T$; (b) $U_{\mathrm{t}} / U_{\mathrm{t}}^{(0)}$ versus $\kappa a$. Solid curves represent the case $Z=1$ and dashed curves denote the case $Z=2$.

\section{CONCLUDING REMARKS}

In this work, the steady-state diffusioosmosis and electroosmosis of a solution of a symmetric electrolyte with a uniformly imposed and arbitrarily oriented concentration gradient or electric field in an ordered array of identical charged cylinders with thin but polarized electric double layers are analyzed using the unit cell model with various boundary conditions at the virtual surface of the cell. On the basis of the assumption of small Peclet and Reynolds numbers, the ionic electrochemical potential and fluid flow fields in the cell were solved analytically and the diffusioosmotic and electroosmotic velocities of the bulk fluid as functions of the porosity of the array of cylinders were obtained in closed-form expressions given by Eqs. [36]-[44] and [51][54]. Comparisons of the results of the cell model with different 
conditions at the outer boundary of the cell have also been provided. For the systems of diffusioosmosis and electroosmosis in a fibrous porous medium discussed in the present work, the relevant experimental data, which are not yet available, would be needed to confirm the validity of each case of the cell model at various ranges of $\zeta e / k T, \kappa a$, and $\varphi$.

Equation [39] for the limiting case of $\varphi=0$ can be used to express the diffusiophoretic and electrophoretic velocities of a single charged circular cylinder in the direction normal to its axis. As to the diffusiophoretic and electrophoretic motions of a cylinder generated by a longitudinal electrolyte concentration gradient or electric field, there is no disturbance in the fluid velocity and electrochemical potential fields caused by the curvature of the cylinder. Thus, the longitudinal velocity of the cylinder is given by Eq. [36] or [54] with $\varphi=0$. For the diffusiophoresis and electrophoresis of a circular cylindrical particle oriented arbitrarily with respect to the imposed electrolyte concentration gradient or electric field, the particle velocity is the vectorial sum of its transverse and longitudinal contributions. For an ensemble of circular cylinders with random orientation, the average diffusiophoretic and electrophoretic velocities (aligned with the direction of the applied field $\nabla n^{\infty}$ or $\mathbf{E}^{\infty}$ ) can be obtained by two thirds of the value given by Eq. [39] (with $\left(\nabla n^{\infty}\right)_{\mathrm{t}}=\left|\nabla n^{\infty}\right|$ and $\left.E_{\mathrm{t}}^{\infty}=\left|\mathbf{E}^{\infty}\right|\right)$ plus one third of the value given by Eq. [36] or [54] (with $\left(\nabla n^{\infty}\right)_{1}=\left|\nabla n^{\infty}\right|, E_{1}^{\infty}=\left|\mathbf{E}^{\infty}\right|$, and $\left.\varphi=0\right)$ (27).

\section{ACKNOWLEDGMENT}

This work was supported by the National Science Council of the Republic of China under Grant NSC90-2214-E-002-019.

\section{REFERENCES}

1. Burgreen, D., and Nakache, F. R., J. Phys. Chem. 68, 1084 (1964).

2. Rice, C. L., and Whitehead, R., J. Phys. Chem. 69, 4017 (1965).
3. Dukhin, S. S., and Derjaguin, B. V., in "Surface and Colloid Science" (E. Matijevic, Ed.), Vol. 7. Wiley, New York, 1974.

4. Prieve, D. C., Anderson, J. L., Ebel, J., and Lowell, M. E., J. Fluid Mech. 148, 247 (1984).

5. Fair, J. C., and Osterle, J. F., J. Chem. Phys. 54, 3307 (1971).

6. Sasidhar, V., and Ruckenstein, E., J. Colloid Interface Sci. 85, 332 (1982).

7. Westermann-Clark, G. B., and Anderson, J. L., J. Electrochem. Soc. 130, 839 (1983).

8. Keh, H. J., and Wu, J. H., Langmuir 17, 4216 (2001).

9. Happel, J., AIChE J. 4, 197 (1958).

10. Happel, J., AIChE J. 5, 174 (1959).

11. Kuwabara, S., J. Phys. Soc. Jpn. 14, 527 (1959).

12. Levine, S., Neale, G. H., and Epstein, N., J. Colloid Interface Sci. 57, 424 (1976).

13. Keh, H. J., and Ding, J. M., J. Colloid Interface Sci. 227, 540 (2000).

14. Levine, S., and Neale, G. H., J. Colloid Interface Sci. 47, 520 (1974).

15. Shilov, V. N., Zharkikh, N. I., and Borkovskaya, Yu. B., Colloid J. USSR (English translation) 43, 434 (1981).

16. Zharkikh, N. I., and Shilov, V. N., Colloid J. USSR (English translation) 43, 865 (1982).

17. Kozak, M. W., and Davis, E. J., J. Colloid Interface Sci. 127, 497 (1989).

18. Ohshima, H., J. Colloid Interface Sci. 188, 481 (1997).

19. Johnson, T. J., and Davis, E. J., J. Colloid Interface Sci. 215, 397 (1999).

20. Ding, J. M., and Keh, H. J., J. Colloid Interface Sci. 236, 180 (2001).

21. Zharkikh, N. I., and Borkovskaya, Yu. B., Colloid J. USSR (English translation) 43, 520 (1982).

22. Kozak, M. W., and Davis, E. J., J. Colloid Interface Sci. 112, 403 (1986).

23. Ohshima, H., J. Colloid Interface Sci. 210, 397 (1999).

24. Lee, E., Lee, Y., Yen, F., and Hsu, J., J. Colloid Interface Sci. 223, 223 (2000).

25. Wei, Y. K., and Keh, H. J., Langmuir 17, 1437 (2001).

26. O’Brien, R. W., J. Colloid Interface Sci. 92, 204 (1983).

27. Keh, H. J., and Chen, S. B., Langmuir 9, 1142 (1993).

28. Levich, V. G., "Physicochemical Hydrodynamics." Prentice Hall, Englewood Cliffs, NJ, 1962.

29. Happel, J., and Brenner, H., "Low Reynolds Number Hydrodynamics." Nijhoff, The Netherlands, 1983.

30. Berryman, J. G., Phys. Rev. A 27, 1053 (1983).

31. Tu, H. J., and Keh, H. J., J. Colloid Interface Sci. 231, 265 (2000).

32. Zukoski, C. F., and Saville, D. A., J. Colloid Interface Sci. 115, 422 (1987). 